\title{
Liquid biopsy and ovarian cancer; Where do we stand?
}

\section{Charalampos Theofanakis, Nikolaos Thomakos, Dimitrios Haidopoulos, Alexandros Rodolakis}

1st Department of Obstetrics \& Gynecology, Alexandra Hospital, National and Kapodistrian University of Athens, Athens, Greece

\section{Correspondence}

Correspondence should be addressed to Charalampos Theofanakis, charalampostheofanakis@yahoo.com

\section{Dear Editor}

Characterized as "silent killer" due to lack of symptoms in the early stages, ovarian cancer represents the most lethal gynecological malignancy. Over the years, there has been an effort to establish effective screening methods in order to assure early detection of the disease. Methods such as serum cancer antigen 125 (CA-125) and transvaginal ultrasound (T/V-US) have been proposed and tested, but with unsatisfactory results, regarding the sensitivity and specificity of the different protocols that are used.

The ongoing need for the development of a cancer biomarker that could help in the early diagnosis of ovarian cancer has led researchers to test the concept of liquid biopsy. Liquid biopsy is a minimally invasive blood-based approach, that could provide information on prognosis, response to treatment, early diagnosis and population screening. The method involves the collection and analysis of circulating tumor cells (CTCs), circulating tumor DNA (ctDNA), circulating cell-free microRNAs, (cfmiRNAs) and exosomes ${ }^{1}$.

CTCs have a crucial role in the hematogenous metastatic spread of epithelial ovarian cancer, andmany studies have attempted to assess the prognostic and predictive potential of these cells. Elevated CTCs in patients during the course of treatment are corre- lated with a significantly shorter progression-free survival (PFS) and overall survival (OS). Moreover, high heterogeneity has been observed by directly measuring gene expression in individual CTCs ${ }^{2}$.

Studies focused on ctDNA in patients with ovarian cancer in order to evaluate its clinical use in terms of early diagnosis, prognosis, response to treatment, while it has also been tested for screening in the general population. This technique encompasses various parameters such as measurement of total ctDNA and/or circulating cell-free mitochondrial DNA quantification, or detection of different genetic and epigenetic alterations, such as chromosomal abnormalities and specific tumor loss of heterozygosity, which are very important in ovarian cancer ${ }^{3}$.

The importance of cfmiRNAs has been investigated by a series of studies, regarding the effectiveness of the method in terms of early diagnosis of ovarian cancer, prognosis, as well as a tool to monitor the response to treatment modalities. Especially regarding early detection, it seems that using a combination of 8 cfmiRNAs, ovarian cancer patients can be distinguished from healthy controls ${ }^{4}$.

Finally, circulating exosomes present with a different profile in ovarian cancer patients, mostly in the 
polarization of tumor-associated macrophages, compared to healthy controls, or patients with benign disease, indicating a possible clinical value these circulating exosomal miRNAs in the early detection of ovarian cancer ${ }^{5}$.

At the moment, liquid biopsy is being investigated through preclinical and clinical trials. Further research on the molecular pathways of epithelial ovarian cancer is needed, so that we can understand the mechanism of resistance to therapies, avoid repeated and unnecessary biopsies and establish a tailored effective treatment, with less cost and fewer side effects for ovarian cancer patients.

\section{References}

1. Giannopoulou, L., et al., Liquid biopsy in ovarian cancer: the potential of circulating miRNAs and exosomes. Transl Res, 2019. 205: p. 77-91.

2. Cheng, X., et al., Circulating cell-free DNA and circulating tumor cells, the "liquid biopsies" in ovarian cancer. J Ovarian Res, 2017.10(1): p. 75.
3. Giannopoulou, L., S. Kasimir-Bauer, and E.S. Lianidou, Liquid biopsy in ovarian cancer: recent advances on circulating tumor cells and circulating tumor DNA. Clin Chem Lab Med, 2018. 56(2): p. 186-197.

4. Yokoi, A., et al., A combination of circulating miRNAs for the early detection of ovarian cancer. Oncotarget, 2017. 8(52): p. 89811-89823.

5. Ying, X., et al., Epithelial ovarian cancer-secreted exosomal miR-222-3p induces polarization of tumor-associated macrophages. Oncotarget, 2016. 7(28): p. 43076-43087.
Received 18-5-2019

Revised 24-5-2019

Accepted 30-5-2019 